\title{
Dieta cetogénica en cáncer: revisión de la literatura
}

\author{
Ketogenic diet in cancer: A literature review \\ Dieta cetogênica no câncer: a revisão da literatura
}

\author{
Karolina Alvarez-Altamirano', Mónica Patricia Bejarano-Rosales' ${ }^{1}$ Erika Areli Rosas-Gonzalez', \\ Karina Miramontes-Balcón ${ }^{2}$, Juan Alberto Serrano-Olvera ${ }^{3}$, Vanessa Fuchs-Tarlovsky ${ }^{1 *}$
}

Recibido: 1 de junio de 2020. Aceptado para publicación: 5 de agosto de 2020

Primero en línea: 5 de septiembre de 2020

https://doi.org/10.35454/rncm.v3n2.168

\section{Resumen}

El cáncer es una problemática de salud pública en el ámbito mundial. El impacto de la dieta sobre el crecimiento y la proliferación tumoral es un área importante de investigación. Las alteraciones propias del metabolismo glucídico en las células con cáncer incluyen la elevación de la dependencia de glucosa y la generación de energía a partir de un proceso anaeróbico. La disminución de la disponibilidad de glucosa en estas células a partir de la dieta surge como alternativa terapéutica para mejorar el curso clínico de pacientes con cáncer. La dieta cetogénica mimetiza los efectos metabólicos del ayuno limitando la disponibilidad de glucosa en el cuerpo; y por lo tanto, podría ser considerada una alternativa viable para mejorar el tratamiento y pronóstico del cáncer.

En esta revisión de la literatura reunimos la evidencia disponible sobre estudios en humanos que permitan describir la forma cómo actúa la dieta cetogénica en pacientes con cáncer, los posibles mecanismos involucrados, así como los estudios en hu manos que puedan respaldar la hipótesis acerca del impacto de la dieta cetogénica en el crecimiento y progresión tumoral.

La evidencia clínica sugiere que la dieta cetogénica es segura en el paciente con cáncer desde un punto de vista nutricional. En vista de la heterogeneidad de la poca evidencia disponible, es necesario que más y mejores estudios clínicos sean llevados a cabo.

Palabras clave: dieta cetogénica, neoplasias, metabolismo.
Abstract

Cancer is a public health problem worldwide. The impact of diet on tumor growth and proliferation is an important area of research. Alterations inherent to glucose metabolism in cancer cells include increased glucose dependence and energy production from an anaerobic process. Decreasing the availability of dietary glucose in these cells emerges as a therapeutic alternative to improve the clinical course of cancer patients. The ketogenic diet mimics the metabolic effects of fasting by limiting the availability of glucose in the body. Therefore, it could be considered a viable alternative to improve the treatment and prognosis of cancer patients.

In this literature review, we collect the available evidence from human studies to describe how the ketogenic diet works in cancer patients and the possible mechanisms involved, as well as human studies that can support the hypothesis of the impact of the ketogenic diet in tumor growth and progression.

Clinical evidence suggests that in cancer patients the ketogenic diet is safe from a nutritional point of view. However, due to the heterogeneity of the little evidence available, more and better clinical studies need to be conducted.

Keywords: Ketogenic diet; Neoplasms; Metabolism.

\section{Resumo}

O câncer é um problema de saúde pública em todo o mundo. O impacto da dieta no crescimento e proliferação de tumores é uma área importante de investigação. As alterações características do metabolismo glicídico nas células cancerígenas incluem a elevação da dependência de glicose e a geração de energia a partir de um processo anaeróbico. Diminuir a disponibilidade de glicose nestas células a partir da dieta surge como uma alternativa terapêutica para melhorar o curso clínico de pacientes com câncer. A dieta cetogênica mimetiza os efeitos metabólicos do jejum, limitando a disponibilidade de glicose no organismo; e, portanto, pode ser uma alternativa viável para melhorar o tratamento e prognóstico do câncer.

Nesta revisão de literatura, reunimos as evidências disponíveis de estudos em humanos que permitam descrever em que consiste a dieta cetogênica no câncer, os possíveis mecanismos envolvidos, bem como os estudos em humanos que podem apoiar a hipótese sobre o impacto da dieta cetogênica no crescimento e progressão tumoral.

Evidências clínicas sugerem que a dieta cetogênica é segura em pacientes com câncer do ponto de vista nutricional. Em vista da heterogeneidade da pouca evidência disponível, é necessário que mais e melhores estudos clínicos sejam realizados.

Palavras-chave: dieta cetogênica, neoplasias, metabolismo. 
Servicio de Nutrición Clínica, Hospital General de México “Dr Eduardo Liceaga", Ciudad de México, México.

2 Servicio de Nutrición Clínica, Hospital General de Ciudad Juárez, México.

3 Centro Médico, ABC, campus observatorio, Ciudad de México, México.

\section{INTRODUCCIÓN}

La dieta cetogénica (DC) se caracteriza por tener una menor proporción de hidratos de carbono $(\mathrm{CHO})$ y una mayor de lípidos, imitando los efectos metabólicos del ayuno y se ha recomendado como parte del tratamiento de algunas enfermedades neurológicas como la epilepsia $^{(1)}$. A inicios del siglo XIX se observó que esta dieta promovía la elevación de cuerpos cetónicos ${ }^{(2)}$. El doctor Russell Wilder de la Clínica Mayo fue quien en 1921 planteó que esta dieta era tan efectiva como el ayuno, por lo que se le atribuyó el término cetogénica. En 1925, el doctor Peterman describió la composición de la DC en niños, la cual consistía en un aporte de $1 \mathrm{~g}$ de proteína por kg de peso corporal, $10 \mathrm{~g}-15 \mathrm{~g}$ de $\mathrm{CHO}$ al día y el resto de las calorías en forma de lípi$\operatorname{dos}^{(3)}$. A pesar de haber mostrado su beneficio en el control de la epilepsia, con el surgimiento de nuevos fármacos antiepilépticos la DC cayó en desuso ${ }^{(1)}$.

El estudio del metabolismo de las células neoplásicas se inició hace un siglo. El conocimiento sobre el papel de la glucosa en el crecimiento tumoral y sobre los cambios adaptativos de las células tumorales ha brindado un importante análisis de la relación tumor - huésped. Considerando la heterogeneidad de la célula tumoral, el "efecto Warburg" es una de las características más importantes y de gran coincidencia en las células cancerígenas, la cual representa un blanco terapéutico susceptible para el tratamiento del cáncer ${ }^{(4)}$. Debido a que este efecto se centra en la generación de recursos energéticos en la célula tumoral por medio de la glicólisis anaeróbica, las modificaciones en los componentes de la dieta, podría ser un área de estudio prometedora como estrategia en la prevención y tratamiento del cáncer ${ }^{(5)}$.

En la última década, la DC en el tratamiento del cáncer ha adquirido mayor interés por un posible efecto en la reducción del crecimiento tumoral, y aumento de la sensibilidad a fármacos. Sin embargo, la evidencia existente proviene de estudios preclínicos y en menor medida estudios en seres humanos. Por ello, su aplicación como tratamiento adyuvante en cáncer aún es cuestionable $^{(6)}$. La siguiente revisión de la literatura
*Correspondencia: Vanessa Fuchs-Tarlovsky vanessafuchstarlovsky@gmail.com

reúne evidencia enfocada en la descripción de la DC, sus mecanismos de acción, efectos potenciales en el tratamiento de cáncer y perspectivas a futuro.

\section{¿QUÉ ES LA DIETA CETOGÉNICA?}

El término DC se refiere a una terapia dietética en la cual a través de la distribución de los macronutrientes que la componen, se produce un estado de cetosis en el cuerpo ${ }^{(7)}$. Se caracteriza por tener un aporte alto de lípidos, bajo en $\mathrm{CHO}$ y adecuado en proteína. La evidencia en la literatura arroja diferentes abordajes de la DC probablemente a que en la actualidad no existe un consenso que describa las características puntuales de este tipo de dieta. En la literatura, la DC tradicional recomienda aportar lípidos/CHO - proteína de 4:1, 3:1 o 2:1. La DC convencional tiene una relación de lípidos a CHO y proteína de 4:1 lo que proporciona una distribución de macronutrientes de aproximadamente $90 \%$ lípidos, $2 \%$ de $\mathrm{CHO}$ y $8 \%$ de proteína, es decir, aporta 4 gramos de lípidos por cada gramo de $\mathrm{CHO}$ proteínas, considerando los requerimientos diarios de proteína del paciente con cáncer de mínimo $1 \mathrm{~g} / \mathrm{kg} / \mathrm{d}$ hasta $1,2-2,0 \mathrm{~g} / \mathrm{kg}$, en comparación con un sujeto sano en quienes se recomienda un consumo diario de $0,8-1 \mathrm{~g} / \mathrm{kg}^{(8)}$.

Otras variaciones de la DC es la rica en Triglicéridos de Cadena Media (TCM), la dieta Atkins Modificada (DAM) y la dieta de bajo índice glicémico (DBIG), son ejemplos de DC, siendo estas últimas dos menos restrictivas (Tabla 1$)^{(9,10)}$.

Una DC enriquecida con TCM proporciona $70 \%$ de grasas, de las cuales los TCM aportan $50 \%$ y $20 \%$ restante a partir de fuentes poliinsaturadas; por lo tanto, permite incluir más $\mathrm{CHO}$ y proteína en la dieta ${ }^{(11)}$. Observando que esta dieta produce un mayor grado de cetosis debido a que la absorción y digestión de lípidos son más rápidas, ya que los TCM pasan directamente del enterocito a la circulación portal, en donde son convertidos en cetonas por el hígado ${ }^{(9)}$. La DAM es más flexible, y no tiene como objetivo la pérdida de peso. Su relación de lípidos/CHO - proteína es de 1:1 
Tabla 1. Distribución de las diferentes propuestas de dietas cetogénicas

\begin{tabular}{|c|c|c|c|c|c|}
\hline \multirow[t]{2}{*}{ Tipo de dieta } & \multirow{2}{*}{$\begin{array}{c}\text { Relación de } \\
\text { lípidos/CHO- } \\
\text { proteína }\end{array}$} & \multicolumn{3}{|c|}{$\begin{array}{l}\text { Porcentaje de } \\
\text { macronutrientes }\end{array}$} & \multirow[t]{2}{*}{ Características generales } \\
\hline & & $\begin{array}{l}\text { Lípidos } \\
\%\end{array}$ & $\begin{array}{c}\mathrm{CHO} \\
\%\end{array}$ & $\begin{array}{l}\text { Proteína } \\
\%\end{array}$ & \\
\hline Dieta cetogénica tradicional & $\begin{array}{l}4: 1 \\
3: 1 \\
2: 1\end{array}$ & $\begin{array}{c}90 \\
85-90 \\
80-85\end{array}$ & $\begin{array}{l}2-4 \\
2-5 \\
5-10\end{array}$ & $\begin{array}{c}6-8 \\
8-12 \\
10-15\end{array}$ & $\begin{array}{l}\text { Se elimina el consumo de frutas y cereales, } \\
\text { por lo que puede ser adecuado suplementar } \\
\text { vitaminas y minerales }\end{array}$ \\
\hline $\begin{array}{l}\text { Dieta rica en triglicéridos de } \\
\text { cadena media }\end{array}$ & $1: 1$ & $60-70$ & $20-30$ & 10 & $\begin{array}{l}\text { TCM }-50 \% \\
\text { Grasas poliinsaturadas } 20 \% \\
\text { Puede causar malestar gastrointestinal }\end{array}$ \\
\hline Dieta Atkins modificada & $1: 1$ & $60-65$ & $5-10$ & $25-35$ & $\begin{array}{l}\text { Iniciar con } 15 \mathrm{~g} \text { de } \mathrm{CHO} \text { al día, y progresar } 5 \mathrm{~g} \\
\text { mensualmente }\end{array}$ \\
\hline Dieta de bajo índice glicémico & $1: 1$ & $60-70$ & $20-30$ & 10 & Alimentos de IG $<50$ \\
\hline
\end{tabular}

CHO: Hidratos de carbono, TCM: Triglicéridos de cadena media, IG: Índice glicémico.

lo que se traduce en una proporción de $65 \%$ de lípidos al día, mientras que el aporte de $\mathrm{CHO}$ inicial es de 15 gy puede progresar $5 \mathrm{~g}$ por mes, hasta llegar a los $30 \mathrm{~g} /$ día, permitiendo cualquier tipo de $\mathrm{CHO}^{(12)}$. La DBIG permite un mayor consumo de $\mathrm{CHO}(40 \mathrm{~g} /$ día - $60 \mathrm{~g}$ / día) suministrados solo en forma de alimentos con un índice glicémico $<50$, evita cambios bruscos en la concentración de insulina y glucosa sérica ${ }^{(10,13)}$.

Algunas recomendaciones extras como son la suplementación de multivitamínicos y el uso de citrato de potasio tienen la finalidad de evitar efectos deletéreos por la prolongada práctica de la $\mathrm{DC}^{(13)}$.

\section{MECANISMOS DE ACCIÓN VINCULADOS A LA DIETA CETOGÉNICA}

Se han descrito distintos factores involucrados en el metabolismo de la célula neoplásica, entre ellos se encuentra la disponibilidad de los nutrientes para el metabolismo, crecimiento y proliferación.

La principal fuente energética de las células sanas y tumorales es la glucosa. La DC desencadena en las células tumorales al efecto Warburg, un fenómeno bioquímico en el que las células cancerosas utilizan en forma predominante la glucólisis en lugar de la fosforilación oxidativa para producir ATP. En consecuencia, la fosforilación oxidativa aparentemente se reduce. Como resultado, las células cancerosas convierten la mayoría de la glucosa entrante en lactato. Aunque la producción de adenosina trifosfato (ATP) es más rápida por glucó- lisis, es menos eficiente en términos de ATP generado por unidad de glucosa consumida que la fosforilación oxidativa, lo cual sugiere una disfunción de la actividad mitocondrial ${ }^{(14)}$. La consecuencia es que las células tumorales requieren una tasa anormalmente más alta de glucosa en comparación con la célula normal. Un alto consumo de glucosa y un aumento de sus transportadores han sido descritos en distintos tipos de cáncer, los cuales corresponden al grado de absorción de la glucosa en tumores agresivos observado en la tomografía por emisión de positrones o $\operatorname{PET}^{(15)}$ (Figura 1).

Además del aumento en la tasa glucolítica de la célula neoplásica, se ha descrito un aumento en la actividad de la ruta pentosas-fosfato, que permite la producción de NADPH, cofactor de los sistemas antioxidantes endógenos glutatión/glutatión peroxidasa $(\mathrm{GPx})$ y la tioredoxina/tioredoxina peroxidasa, y mantiene el estado de óxido-reducción de la célula tumoral. En algunos tipos de cáncer de mama agresivos estudiados en modelos animales han mostrado la relación de un aumento en la producción de GPx y la resistencia de fármacos antineoplásicos por el efecto que ejerce GPx en el balance redox de la célula neoplásica ${ }^{(16)}$. La disminución del estímulo de la ruta glucolítica en estudios in vivo e in vitro resulta de interés en las terapias biológicas contra el cáncer con lo cual existe un menor suministro de glucosa, menor sustrato de la vía de las pentosas fosfato; y por lo tanto, un daño prooxidativo selectivo de la célula neoplásica ${ }^{(17)}$. Aunado a lo anterior, la disminución prolongada de glucosa en 


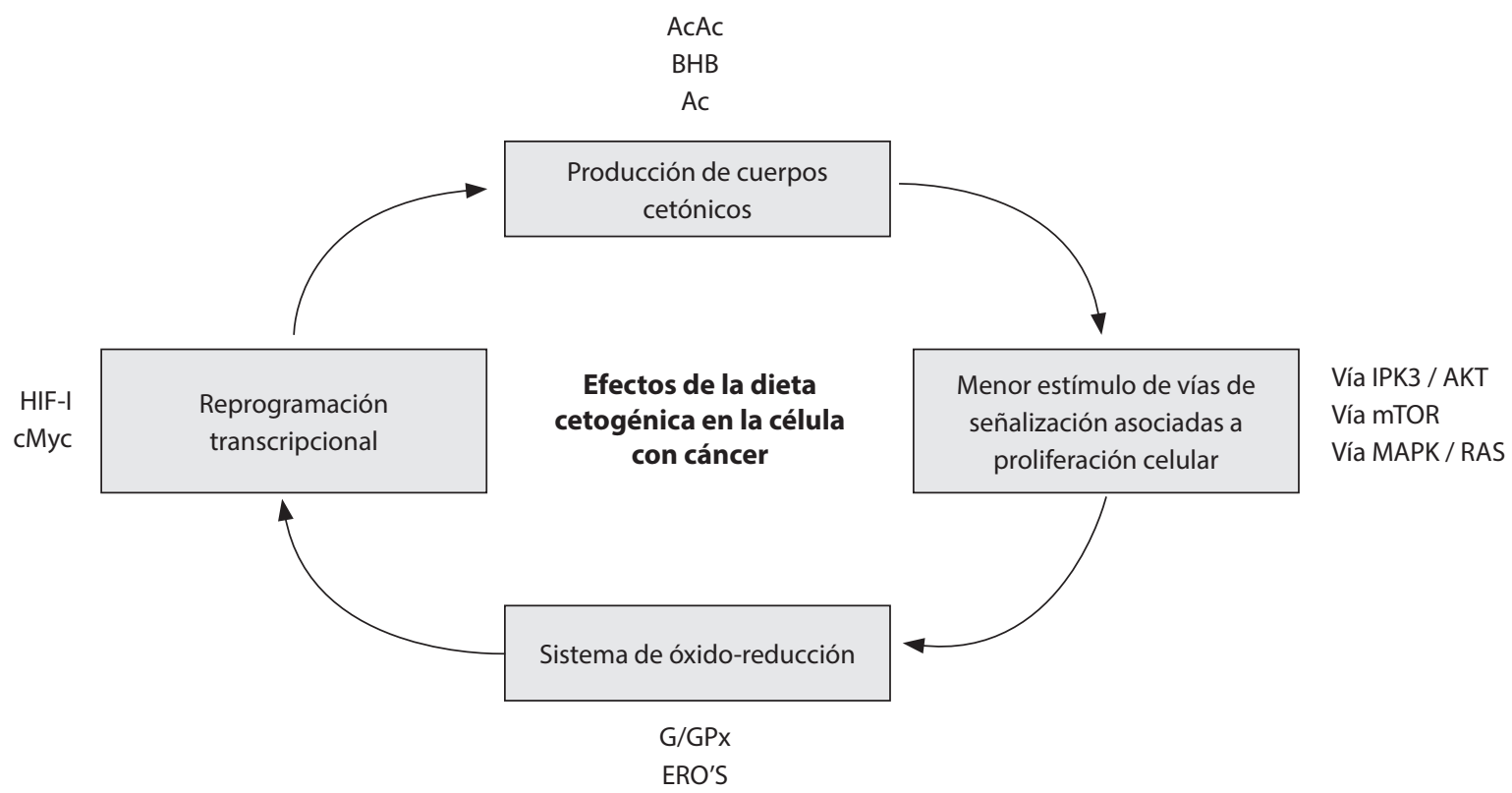

Figura 1. Mecanismos de acción asociado al efecto de la dieta cetogénica (DC) en cáncer. La disminución de la disponibilidad de la glucosa por medio de la DC condiciona múltiples estímulos celulares. Existe un menor estímulo de la insulina y receptores de hormonas disminuyendo la actividad de vías de crecimiento y proliferación celular como fosfoinositol-3-quinasa (IPK3)/ proteína quinasa B (AKT), diana de rapamicina en células de mamíferos (mTOR) y proteína quinasas activadas por mitógenos (MAPK)/ genes RAS. Los sistemas de óxido reducción de la célula se ven afectados al disminuir el sustrato de la glucólisis destinado a la vía de las pentosas fosfato (fructosa-1.6-difosfato) condicionando el balance redox de la célula tumoral por disminución de la actividad de glutatión (G)/ glutatión peroxidasa (GPx). La incrementada producción de lactato en presencia de concentraciones bajas de oxígeno promueve la adaptación celular por estímulo de Factor Inducible por Hipoxia-1 (HIF-1) y factor transcripción cMyc, quienes inhiben de forma secundaria la fosforilación oxidativa y general un aumento en la conversión de piruvato-lactato. Como efecto metabólico, la DC permite la producción de los cuerpos cetónicos: acetoacetato (AcAc), $\beta$-hidroxibutirato (BHB) y acetato (Ac), que sirven como intermediarios de los sustratos para el ciclo de creebs como activadores de receptores acoplados a proteínas $\mathrm{G}$ de membrada con actividad antitumoral. Otras abreviaciones ERO: especies reactivas de oxígeno.

el huésped conduce a las células normales a un estado de cetosis, el cual reduce la disponibilidad de glucosa en la células neoplásicas, limitando así su crecimiento, mientras las células normales se adaptan a la utilización de los cuerpos cetónicos como fuente energética al inactivar la vía insulina/lipoproteína lipasa sensible a hormonas y activación del glucagon ${ }^{(18)}$.

En presencia de ayuno o una dieta baja en $\mathrm{CHO}$ tradicional (4:1 o la DAM), el organismo genera un aumento de los cuerpos cetónicos utilizados como sustrato energético, que principalmente son el acetoacetato (AcA) y b-hidroxibutirato (BHB) ${ }^{(19)}$. Tras las primeras 72 horas de inicio de la DC se observa un aumento significativo en la concentración de los cuerpos cetónicos con una meseta aproximada hasta el día $30^{(20)}$. La elevación de los cuerpos cetónicos ha mostrado efectos anti proliferativos en los colonocitos por la activación del receptor GPR109A, de niacina/butirato ${ }^{(21)}$.
En células sanas la glucosa estimula la liberación de la insulina por el páncreas promueve la interacción de los receptores de la hormona del crecimiento y las hormonas de crecimiento para producir el factor de crecimiento similar a la insulina 1 (IGF-1) en el hígado. Esto se ha relacionado con un aumento del riesgo de cáncer en sujetos sanos y con obesidad, además de que en pacientes oncológicos promueve el crecimiento y la proliferación celular vía IGF-1/PI3K/AKT/ diana de rapamicina en mamíferos (mTOR) ${ }^{(22,23)}$. De esta forma, la restricción de glucosa se ha asociado a la prevención de cáncer y la progresión del mismo; sin embargo, en modelos animales no ha sido suficiente para evitar la progresión tumoral. Las dietas cetogénicas también amplifican la proteína quinasa activada por monofosfato de adenosina (por sus siglas en inglés, AMPK), que inhibe la glucólisis aeróbica y suprime la proliferación, invasión y migración tumoral. Los modelos de 
ratón con cáncer metastásico muestran que las cetonas exógenas tienen efectos citotóxicos directos sobre la viabilidad del tumor ${ }^{(9)}$.

Un elemento que puede ser contrarrestado es la expresión de lactato, la cual está relacionada con la oxidación de NADH A NAD+, y con una disminución del estímulo del receptor GPR81 del lactato. La actividad de este receptor está asociada a la proliferación celular, angiogénesis y quimio resistencia ${ }^{(24)}$.

Otros factores involucrados en el metabolismo glucolítico de la célula neoplásica son los oncogenes HIF-1, c-Myc, principalmente producidos por los niveles altos de lactato. Los dos primeros están implicados en la expresión de la enzima lactato deshidrogenasa (LDH) y piruvato deshidrogenasa quinasa 1 (por sus siglas en inglés, PDK1 ${ }^{(23)}$. La LDH está involucrada en la conversión de piruvato-lactato en el citosol y la PDK1 en la inhibición de la enzima piruvato deshidrogenasa, reduciendo la entrada de piruvato al ciclo tricarboxílico, condicionando la función mitocondrial. En este sentido la DC o la restricción calórica parecen generar no solo cambios en los sustratos metabólicos de la célula tumoral sino también en la señalización nuclear. En modelos animales de glioma se ha relacionado con la supresión de HIF-1, y del receptor VEGF-2 reduciendo la microvasculatura del tumor ${ }^{(25)}$.

\section{EVIDENCIA DEL USO DE LA DIETA CETOGÉNICA EN PACIENTES CON CÁNCER}

Esta evidencia derivada de estudios preclínicos, muestra un uso potencial de esta dieta como terapia antineoplásica, principalmente en modelos de glioblastoma ${ }^{(9)}$, glioma $^{(26)}$, páncreas ${ }^{(27)}$ y colon ${ }^{(28)}$, obteniendo mayor rango de supervivencia e incluso la reversión de la caquexia. En cambio, la DC parece no presentar un efecto similar en modelos renales ya que se ha descrito un efecto contrario al esperado con disminución de la sobrevida acompañado de pérdida de peso $^{(9)}$.

En consecuencia, es necesario tener en cuenta la variabilidad de los resultados y los posibles beneficios no deben generalizarse, ya que aparentemente, según el tipo de cáncer, las células responden de manera distinta a la DC. En humanos existe poca evidencia de los beneficios potenciales de la DC en pacientes adultos oncológicos. En esta revisión utilizando criterios de búsqueda como "dieta cetogénica" o "dieta que imita el ayuno" o "dieta baja en carbohidratos” y "cáncer" o "malignidad”, buscamos artículos en estudios en humanos, escritos en inglés a partir del año 2000 a la fecha y encontramos
11 artículos que reportan efectos de la DC en este tipo de pacientes. Estos estudios se han realizado en poblaciones heterogéneas, y con un número de participantes muy limitado que en la mayoría de los casos presentan un abandono de la dieta.

La evidencia reportada está basada en diseños de estudios de baja evidencia científica: reportes y series de casos, estudios observacionales y pocos estudios clínicos. El tamaño de la muestra es pequeño en la mayoría de los casos, desde 1 persona hasta 129 pacientes, y con diferentes objetivos en la implementación de la $\mathrm{DC}^{(29-39)}$. Un punto a resaltar es la dificultad de la adherencia a la dieta, así como los periodos de seguimiento. Estos últimos son variables y se extienden en algunos casos, de 2 a 31 meses, situación que genera dificultad al comparar el efecto del manejo con DC. En la Tabla 2, se pueden apreciar los estudios incluídos en esta revisión.

En cuanto a los hallazgos, la DC parece ser segura en pacientes con cáncer ya que no se reportan efectos adversos severos, datos de toxicidad con los tratamientos antineoplásicos acorde a la Common Terminology Criteria for Adverse Events ${ }^{(32)}$ o aumento en la progresión en comparación con grupos controles, en aquellos estudios que los reportan ${ }^{(31,34,38)}$. Dentro de los casos que reportan sintomatología relacionada con la $\mathrm{DC}$, se trata de síntomas leves como constipación o fatiga, hambre durante la primera semana de la aplicación de la dieta ${ }^{(30)}$, y disminución de la percepción de la calidad de vida en tres $\operatorname{casos}^{(31)}$. En otros casos los estudios no especifican si la falta de apego/abandono de la dieta se relaciona con algún efecto adverso leve o severo. Ningún estudio reporta algún efecto grave secundario a la DC (como en el caso de hipoglucemia o desmayos), $y$ en general los pacientes reportan una "buena” tolerancia a la intervención con la DC.

La información sobre la pérdida de peso es controversial, algunos estudios reportan que los pacientes mantienen el peso, en especial el tejido magro, durante la intervención y otros que puede presentarse disminución del peso sin afectar la progresión de la enfer$\operatorname{medad}^{(34,35,38)}$. Desde el punto de vista nutricional es interesante puesto que los pacientes con cáncer tienden a presentar una pérdida de peso involuntaria durante los tratamientos médicos.

El objetivo perseguido por los estudios es distinto; y por lo tanto, llegar a conclusiones sobre los efectos en el tratamiento antineoplásico es difícil. Los estudios muestran resultados tanto a favor como con efecto nulo, y principalmente realiza evaluación posterior a la inter- 
Tabla 2. Estudios en humanos que reportan el efecto de la Dieta Cetogénica en el crecimiento o progresión tumoral

\begin{tabular}{|c|c|c|c|c|c|c|c|c|c|}
\hline $\begin{array}{l}\text { Autor } \\
\text { (año) }\end{array}$ & Diseño & $\begin{array}{c}\text { Muestra } \\
(\mathrm{n}=)\end{array}$ & Tipo de cáncer & $\begin{array}{l}\text { Tratamiento } \\
\text { recibido }\end{array}$ & $\begin{array}{l}\text { Composición } \\
\text { dieta }\end{array}$ & Duración & Objetivo & Resultados & Referencia \\
\hline $\begin{array}{l}\text { Zuccoli y } \\
\text { col. (2010) }\end{array}$ & $\begin{array}{l}\text { Reporte de } \\
\text { caso }\end{array}$ & 1 & $\begin{array}{l}\text { Glioblastoma } \\
\text { multiforme }\end{array}$ & $\mathrm{RT}+\mathrm{QT}$ & $\begin{array}{l}\text { 4:1 grasas: } \mathrm{CHO} \\
+ \text { proteína) }+ \\
\text { vitaminas y } \\
\text { minerales }\end{array}$ & $\begin{array}{l}8 \text { semanas } \\
+5 \text { meses } \\
\text { posteriores } \\
\text { con dieta baja } \\
\text { en calorías }\end{array}$ & $\begin{array}{l}\text { Glucosa sérica } \\
\text { y reducción de } \\
\text { peso }\end{array}$ & $\begin{array}{l}\text { Recurrencia del tumor } 10 \text { meses } \\
\text { después del tratamiento. } \\
\text { Reducción en niveles de } \\
\text { glucosa } \\
\text { Reducción de } 20 \% \text { de peso. }\end{array}$ & 29 \\
\hline $\begin{array}{l}\text { Schmidt y } \\
\text { col. }(2011)\end{array}$ & Observacional & 16 & $\begin{array}{l}\text { Tumores } \\
\text { metastásicos } \\
\text { avanzados de } \\
\text { ovario, mama, } \\
\text { parótida, cáncer, } \\
\text { tiroides, colon, } \\
\text { endometrio, } \\
\text { pulmón, } \\
\text { estómago, esófago }\end{array}$ & $\begin{array}{l}\text { Tx previo a la } \\
\text { dieta de Qx, RT, } \\
\text { QT }\end{array}$ & $\begin{array}{l}<70 \text { g de } \mathrm{CHO} \text { al } \\
\text { día }+ \text { Mezcla de } \\
\text { proteínas y grasa }\end{array}$ & 12 semanas & EORTC QLQ - C30 & $\begin{array}{l}\text { Mejoró el aspecto emocional, } \\
\text { disminuyó el insomnio } \\
\text { Reducción significativa de peso, } \\
\text { y colesterol HDL y LDL }\end{array}$ & 30 \\
\hline $\begin{array}{l}\text { Rieger y } \\
\text { col. (2014) }\end{array}$ & $\begin{array}{l}\text { Ensayo clínico } \\
\text { piloto }\end{array}$ & 17 & Glioblastoma & $\begin{array}{l}\geq 6 \text { meses } \\
\text { después de la } \\
\text { cirugía } \\
y \geq 3 \text { meses } \\
\text { después de RT/QT }\end{array}$ & $\begin{array}{l}\text { Máximo } 60 \mathrm{~g} \\
\text { de CHO / día. } \\
\text { Productos } \\
\text { complementarios } \\
\text { dietéticos de } \\
\text { Tavarin }{ }^{\oplus}\end{array}$ & 6 - 8 semanas & $\begin{array}{l}\text { Glucosa en } \\
\text { sangre y } \mathrm{HbA} 1 \mathrm{c}, \\
\mathrm{HDL}, \mathrm{LDL}, \\
\text { triglicéridos }\end{array}$ & $\begin{array}{l}\text { No se produjeron cambios } \\
\text { significativos en los parámetros } \\
\text { de laboratorio }\end{array}$ & 31 \\
\hline $\begin{array}{l}\text { Champ y } \\
\text { col. (2014) }\end{array}$ & $\begin{array}{l}\text { Observacional } \\
\text { retrospectivo }\end{array}$ & 6 & $\begin{array}{l}\text { Glioblastoma } \\
\text { multiforme }\end{array}$ & RTQ & $50 \mathrm{~g}$ de $\mathrm{CHO}$ al día & 5-20 meses & $\begin{array}{l}\text { Niveles de } \\
\text { glucosa }\end{array}$ & $\begin{array}{l}\text { Reducción de glucosa } 122 \\
\text { vs } 84 \mathrm{mg} / \mathrm{dl} \text {. }\end{array}$ & 32 \\
\hline $\begin{array}{l}\text { Branca } \\
\text { JJV, y col. } \\
(2015)\end{array}$ & $\begin{array}{l}\text { Reporte de } \\
\text { caso }\end{array}$ & 1 & $\begin{array}{l}\text { Cáncer de mama: } \\
\text { HER2 (+), RE (+), } \\
\text { KI67 (+). }\end{array}$ & Cirugía & $\begin{array}{l}\text { Dieta cetogénica } \\
\text { estricta (no } \\
\text { menciona } \\
\text { distribución) } \\
+10,000 \text { Ul de } \\
\text { Vitamina D3. }\end{array}$ & 3 semanas & $\begin{array}{l}\text { Determinación } \\
\text { diagnóstica y } \\
\text { resección mama } \\
\text { derecha. }\end{array}$ & $\begin{array}{l}\text { HER2 }(+) \text { tras la biopsia. } \\
\text { HER2 }(-) \text { tras análisis de } \\
\text { mastectomía derecha. RE (+), } \\
\text { KI67 (+). }\end{array}$ & 33 \\
\hline $\begin{array}{l}\text { Tan } \\
\text { Shalaby y } \\
\text { col. (2016) }\end{array}$ & Observacional & 17 & $\begin{array}{l}\text { Múltiples tipos de } \\
\text { cáncer }\end{array}$ & Sin QT & $\begin{array}{l}20 \text { - } 40 \text { g de } \mathrm{CHO} \\
\text { al día }\end{array}$ & 16 semanas & $\begin{array}{l}\text { Seguridad y } \\
\text { factibilidad }\end{array}$ & $\begin{array}{l}\text { Mejoró calidad de vida. } \\
\text { Los pacientes que } \\
\text { disminuyeron al menos el } 10 \% \\
\text { de su peso mejoraron. }\end{array}$ & 34 \\
\hline
\end{tabular}


Tabla 2. Estudios en humanos que reportan el efecto de la Dieta Cetogénica en el crecimiento o progresión tumoral (continuación)

\begin{tabular}{|c|c|c|c|c|c|c|c|c|c|}
\hline $\begin{array}{l}\text { Autor } \\
\text { (año) }\end{array}$ & Diseño & $\begin{array}{c}\text { Muestra } \\
(\mathrm{n}=)\end{array}$ & Tipo de cáncer & $\begin{array}{l}\text { Tratamiento } \\
\text { recibido }\end{array}$ & $\begin{array}{c}\text { Composición } \\
\text { dieta }\end{array}$ & Duración & Objetivo & Resultados & Referencia \\
\hline $\begin{array}{l}\text { Klement y } \\
\text { Sweeney } \\
(2016)\end{array}$ & Serie de casos & 6 & Cáncer avanzado & RTQ & $\begin{array}{l}<50 \mathrm{~g} \text { de } \mathrm{CHO} \text { al } \\
\text { día } \\
\text { 2:1 (grasas: } \mathrm{CHO}+ \\
\text { proteína) }\end{array}$ & 32-73 días & $\begin{array}{l}\text { EORTC QLQ-C30, } \\
\text { composición } \\
\text { corporal, } \\
\text { regresión tumoral }\end{array}$ & $\begin{array}{l}\text { Todos los pacientes } \\
\text { disminuyeron su peso, se } \\
\text { preservó la masa muscular. } \\
\text { Regresión tumoral en cinco } \\
\text { pacientes }\end{array}$ & 35 \\
\hline $\begin{array}{l}\text { Artzi y col. } \\
(2017)\end{array}$ & Observacional & 9 & Glioblastoma & $\begin{array}{l}\text { Anticuerpo } \\
\text { monoclonal }\end{array}$ & 4:1 (grasas: $\mathrm{CHO}$ ) & $2-31$ meses & $\begin{array}{l}\text { Caracterizar } \\
\text { cambios en } \\
\text { metabolitos } \\
\text { cerebrales }\end{array}$ & $\begin{array}{l}\text { Es incierto si existe acumulación } \\
\text { de cuerpos cetónicos a nivel } \\
\text { cerebral o disminuye su } \\
\text { utilización }\end{array}$ & 36 \\
\hline $\begin{array}{l}\text { Tóth y col. } \\
(2017)\end{array}$ & $\begin{array}{l}\text { Reporte de } \\
\quad \text { caso }\end{array}$ & 1 & Rectal & RT & 2:1 (grasas: $\mathrm{CHO}$ ) & 22,5 meses & $\begin{array}{l}\text { Sin objetivos } \\
\text { generales o } \\
\text { específicos } \\
\text { definidos }\end{array}$ & $\begin{array}{l}\text { Se pospuso la cirugía dos años. } \\
\text { La enfermedad progresó. }\end{array}$ & 37 \\
\hline $\begin{array}{l}\text { Cohen y } \\
\text { col. }(2018)\end{array}$ & Ensayo clínico & 45 & $\begin{array}{l}\text { Endometrio } \\
\text { Ovario }\end{array}$ & QT & $\begin{array}{l}\text { Aproximadamente } \\
70 \% \text { grasa, hasta } \\
25 \% \text { proteína, } \\
\text { y } 5 \% \text { CHO }(\leq 20 \\
\text { g / d) }\end{array}$ & 12 semanas & $\begin{array}{l}\text { Lípidos séricos, } \\
\text { pérdida de peso }\end{array}$ & $\begin{array}{l}\text { Sin diferencias significativas } \\
\text { entre los grupos }\end{array}$ & 38 \\
\hline \begin{tabular}{l|} 
de Groot y \\
col. \\
$(2020)$
\end{tabular} & $\begin{array}{l}\text { Ensayo clínico } \\
\text { multicéntrico }\end{array}$ & 129 & Cáncer de mama & $\begin{array}{l}\text { QT y RT } \\
\text { neoadyuvante }\end{array}$ & $\begin{array}{l}\text { No específica } \\
\text { composición }\end{array}$ & $\begin{array}{l}2-4 \text { ciclos de } \\
\text { tratamiento }\end{array}$ & \begin{tabular}{|l|} 
Toxicidad y \\
respuesta al \\
tratamiento. \\
Efectos \\
metabólicos y en \\
la calidad de vida
\end{tabular} & $\begin{array}{l}\text { Sin diferencias en toxicidad. } \\
3 \text { veces mayor respuesta a la RT. } \\
\uparrow \text { Cuerpos cetónicos, } \downarrow \text { niveles } \\
\text { de glucosa, insulina, IGF-1. } \\
\text { Sin cambios en toxicidad por } \\
\text { QT. } \\
\downarrow \text { Daños al ADN de linfocitos } \\
\text { posterior a la QT. }\end{array}$ & 39 \\
\hline
\end{tabular}

ACS: American Cancer Society Diet, RT: radioterapia; QT: quimioterapia, CX: cirugía, CHO: carbohidratos, RE: receptor de estrógenos, KI67: antígeno Ki-67, HER2 (por sus síglas en inglés): receptor del factor de crecimiento epidérmico 2, EORTC QLQ-C30: European Organisation for Research and Treatment of Cancer Quality of Life Questionnaire C-30. 
vención con la DC. Dos de tres estudios de caso con un paciente cada uno (uno con diagnóstico de glioblastoma y otro con cáncer de recto) indican la detección de recurrencia del cáncer posterior a la suspensión de la DC. Ambos casos tuvieron una adherencia a la dieta entre 22 y 28 semanas y tras una corta reducción en la adherencia a la dieta reportaron la recurrencia de cáncer o retraso en la indicación de cirugía ${ }^{(29,37)}$. Otro reporte de caso de una paciente con cáncer recurrente de mama, con positividad al marcador del receptor de estrógenos y HER2, observó en forma inesperada que posterior a la mastectomía realizada, la expresión del HER2 presentó un resultado negativo. Esta paciente recibió una intervención con DC estricta durante 3 semanas $+10,000$ UI de vitamina D3 por decisión propia, entre el periodo del diagnóstico y la cirugía $^{(33)}$. En los tres casos no reportan efectos adversos.

La evidencia más basta surge de tres estudios clínicos realizados en glioblastoma, tumores ginecológicos y cáncer de mama ${ }^{(31,38,39)}$. De estos, 17 de 20 pacientes con glioblastoma accedieron a realizar la DC entre 6 y 8 semanas. Se evaluaron los niveles sanguíneos de glucosa, HDL, LDL y triglicéridos, y la recurrencia; sin embargo, no se presentaron cambios significativos en ningún parámetro ya que algunos pacientes abandonaron la dieta entre la semana 6 y 8 , y solo uno concluyó las 8 semanas $^{(31)}$. Otro estudio clínico en pacientes con cáncer ginecológico (endometrio y ovario) recolectó e hizo seguimiento a 45 mujeres por 12 semanas con una dieta más restrictiva que el estudio anterior y observaron los cambios en la composición corporal por medio de una absorciometría dual por rayos X (DXA) y parámetros metabólicos. Los resultados de este estudio muestran que las pacientes con DC redujeron de manera significativa su masa grasa visceral y masa grasa, y mantuvieron la muscular. Este efecto se atribuyó a la elevación de cuerpos cetónicos, específicamente del BHB, acompañado de una disminución de IGF-1 ${ }^{(38)}$.

El estudio clínico aleatorizado más reciente se trata de un estudio multicéntrico realizado en 129 pacientes con cáncer de mama. Las pacientes recibieron quimioterapia (QT) neoadyuvante y radioterapia (RT) con la finalidad de valorar la toxicidad, respuesta al tratamiento; además, de efectos metabólicos y de calidad de vida. Este estudio multicéntrico publicado en una revista indexada, y con una mayor muestra $(\mathrm{n}=129)$ de pacientes, presenta la desventaja de no indicar la composición de la dieta. Este estudio hace referencia al uso de una dieta que imita los efectos del ayuno (DIEA) versus una dieta convencional. Los investigadores hicieron un seguimiento de los pacientes en relación con los ciclos de quimioterapia tolerados o indicados, de los cuales llevaron a cabo la intervención de la dieta durante 2 - 4 ciclos de tratamiento médico. Los resultados indican que los pacientes con la dieta tipo DIEA no presentaron diferencias en toxicidad a la QT, pese a que en este grupo no se utilizó tratamiento paralelo con glucocorticoides. Además de esto presentaron mayor tolerancia a la RT, acompañado del aumento de los cuerpos cetónicos, disminución de los niveles de glucosa, IGF-1 e insulina ${ }^{(39)}$.

\section{CONCLUSIÓN}

Teniendo en cuenta la evidencia científica actual y en particular la proveniente de estudios clínicos en seres humanos, aún es cuestionable la aplicación de la dieta cetogénica como tratamiento adyuvante en cáncer.

Pese a la evidencia existente en estudios preclínicos, e incluso ya aplicada a algunos animales de experimentación, no consideramos que sea suficiente para que los profesionales de la salud recomienden este tipo de dietas en pacientes oncológicos, y mucho menos que los pacientes se autoprescriban sin supervisión de un profesional de la salud.

La necesidad de conducir más y mejores estudios clínicos en humanos que demuestren el potencial beneficio de la DC en el paciente con cáncer requiere la integración del nutriólogo clínico, quien en colaboración con el médico oncólogo desarrollan este campo de la investigación. Un abordaje dietético ajustado a las necesidades y la situación clínica particular de los pacientes es importante para lograr un buen apego al tratamiento, y por lo tanto, a resultados más consistentes.

\section{Financiación}

Los autores no declaran financiamiento relacionado con la elaboración o publicación del presente trabajo.

\section{Declaración de conflicto de intereses}

Los autores no declaran conflicto de intereses.

\section{Declaración de autoría}

AAK redactó el manuscrito. AAK, BRMP y GREA reunieron la información y analizaron la información. $\mathrm{MK}$ y SA contribuyeron al desarrollo del protocolo. FV revisó críticamente el artículo. Todos los autores validaron la versión final. 


\section{Referencias bibliográficas}

1. Wheless JW. History of the ketogenic diet. Epilepsia. 2008;49(Suppl 8):3-5. doi: https://doi.org/10.1111/j.15281167.2008.01821.x.

2. Geyelin HR. Fasting as a method for treating epilepsy. Med Rec. 1921. 99:1037-9.

3. Peterman MG. The ketogenic diet in epilepsy. JAMA: The Journal of the American Medical Association. 1979;84(26). doi:10.1001/jama.1925.02660520007003.

4. Warburg O, Dickens F. The metabolism of tumors. Publisher: Constable, London, 1930.

5. Hanahan D, Weinberg RA. Hallmarks of Cancer: The Next Generation. Cell. 2011;144(5):646-74. doi: https://doi. org/10.1016/j.cell.2011.02.013.

6. Sremanakova J, Sowerbutts AM, Burden S. A systematic review of the use of ketogenic diets in adult patients with cancer. J Hum Nutr Diet. 2018; 31(6):793-802. doi: https://doi. org/10.1111/jhn.12587.

7. Weber DD, Aminazdeh-Gohari S, Kofler B. Ketogenic diet in cancer therapy. Aging. 2018;10(2):164-5. doi: https://doi. org/10.18632/aging.101382.

8. Arends J, Bachmann P, Baracos V, Barthelemy N, Bertz H, Bozzetti $\mathrm{F}$, et al. ESPEN guidelines on nutrition in cancer patients. Clin Nutr. 2017; 36(1):11-48. doi:https://doi. org/10.1016/j.clnu.2016.07.015.

9. Weber DD, Aminzadeh-Gohari S, Tulipan J, Catalano L, Feichtinger RG, Kofler B. Ketogenic diet in the treatment of cancer-Where do we stand? Mol Metab. 2020; 33:102-21. doi: https://doi.org/10.18632/aging.101382.

10. Zupec-Kania BA, Spellman E. An Overview of the Ketogenic Diet for Pediatric Epilepsy. Nutr Clin Pract. 2008; 23(6):58996. doi: https://doi.org/10.1177/0884533608326138.

11. Kossoff EH, Wang HS. Dietary Therapies for Epilepsy. Biomed J. 2013;36(1):2-8. doi: https://doi.org/10.4103/23194170.107152 .

12. Kossoff EH, Dorward JL. The Modified Atkins Diet. Epilepsia. 2008; 49(Suppl. 8):37-41. doi: https://doi.org/ 10.1111/j.1528-1167.2008.01831.x.

13. Klement RJ, Kämmerer U. Is there a role for carbohydrate restriction in the treatment and prevention of cancer? Nutr Metab. 2011;8(1):75. doi: https://doi.org/10.1186/17437075-8-75.

14. Herrera-González NE, Martínez-García F, Mejía-Jiménez E. El efecto Warburg: la mano derecha en el desarrollo del cáncer. Rev Esp Méd Quir. 2015; 20(2):171-7.

15. Adekola K, Rosen ST, Shanmugam M. Glucose transporters in cancer metabolism. Curr Opin Oncol. 2012; 24(6):650-4. doi: https://doi.org/10.1097/CCO.0b013e328356da72.

16. Buettner GR. Superoxide dismutase in redox biology: the roles of superoxide and hydrogen peroxide. Anticancer Agents Med Chem. 2011;11(4):341-6. doi: https://doi. org/10.2174/187152011795677544.
17. Hegedűs C, Kovács K, Polgár Z, Regdon Z, Szabó É , Robaszkiewicz A, et al. Redox control of cancer cell destruction. Redox Biol. 2018;16:59-74. doi: https://doi. org/10.1016/j.redox.2018.01.015.

18. Klement RJ. The emerging role of ketogenic diets in cancer treatment. Curr Opin Clin Nutr Metab Care. 2019; 22(2): 12934. doi: https://doi.org/10.1097/MCO.0000000000000540.

19. Glew RH. You can get there from here: acetone, anionic ketones and even-carbon fatty acids can provide substrates for gluconeogenesis. Niger J Physiol Sci. 2010; 25(1):2-4.

20. Veech RL. The therapeutic implications of ketone bodies: the effects of ketone bodies in pathological conditions: ketosis, ketogenic diet, redox states, insulin resistance, and mitochondrial metabolism. Prostaglandins Leukot Essent Fatty Acids. 2004;70(3):309-19. doi: https://doi.org/10.1016/j. plefa.2003.09.007.

21. Rahman M, Muhammad S, Khan MA, Chen H, Ridder DA, Müller-Fielitz $\mathrm{H}$, et al. The beta-hydroxybutyrate receptor HCA2 activates a neuroprotective subset of macrophages. Nature Commun. 2014; 5:3944. doi: https://doi. org/10.1038/ncomms4944.

22. Goncalves MD, Hopkins BD, CantleyLC. Phosphatidylinositol 3-kinase, growth disorders, and cancer. N Engl J Med. 2018;379(21):2052-62. doi: https://doi.org/10.1056/ NEJMra1704560.

23. Yin C, Qiea S, Sang N. Carbon Source Metabolism and Its Regulation in Cancer Cells. Crit Rev Eukaryot Gene Expr. 2012;22(1):17-35. doi: https://doi.org/10.1615/critreveukargeneexpr.v22.i1.20.

24. Ristic B, Bhutia YD, Ganapathy V. Cell-surface G-proteincoupled receptors for tumor-associated metabolites: A direct link to mitochondrial dysfunction in cancer. Biochim Biophys Acta Rev Cancer. 2017;1868(1):246-57. doi: https://doi. org/10.1016/j.bbcan.2017.05.003.

25. Woolf EC, Curley KL, Liu Q, Turner GH, Charlton JA, Preul $\mathrm{MC}$, et al. The ketogenic diet alters the hypoxic response and affects expression of proteins associated with angiogenesis, invasive potential and vascular permeability in a mouse glioma model. PLoS One. 2015; 10(6): e0130357. doi: https://doi. org/10.1371/journal.pone.0130357.

26. Shukla SK, Gebregiworgis T, Purohit V, Chaika NV, Gunda V, Radhakrishnan $\mathrm{P}$, et al. Metabolic reprogramming induced by ketone bodies diminishes pancreatic cancer cachexia. Cancer Metab. 2014;2:18. doi: https://doi.org/10.1186/2049-30022-18.

27. Tisdale MJ, Brennan RA, Fearon KC. Reduction of weight loss and tumour size in a cachexia model by a high fat diet. Br J Cancer. 1987; 56(1):39-43. doi: https://doi.org/10.1038/ bjc.1987.149.

28. BeckSA, Tisdale MJ. Effect of insulin on weight loss and tumour growth in a cachexia model. Br J Cancer. 1989;59(5):677-81. doi: https://doi.org/10.1038/bjc.1989.140.

29. Zuccoli G, Marcello N, Pisanello A, Servadei F, Vaccaro S, Mukherjee P, et al. Metabolic management of glioblastoma 
multiforme using standard therapy together with a restricted ketogenic diet: Case Report. Nutr Metab. 2010;7:33. doi: https://doi.org/10.1186/1743-7075-7-33.

30. Schmidt M, Pfetzer N, Schwab M, Strauss I, Kämmerer U. Effects of a ketogenic diet on the quality of life in 16 patients with advanced cancer: A pilot trial. Nutr Metab. 2011;8(1):54. doi: https://doi.org/10.1186/1743-7075-8-54.

31. Rieger J, Bähr O, Maurer GD, Hattingen E, Franz K, Brucker D, et al. ERGO: a pilot study of ketogenic diet in recurrent glioblastoma. Int J Oncol. 2014;44(6):1843-52. doi: https:// doi.org/10.3892/ijo.2014.2382.

32. Champ CE, Palmer JD, Volek JS, Werner-Wasik M, Andrews DW, Evans JJ, et al. Targeting metabolism with a ketogenic diet during the treatment of glioblastoma multiforme. J Neurooncol. 2014;117(1):125-31. doi: https://doi.org/ 10.1007/s11060-014-1362-0.

33. Branca JJV, Pacini S, Ruggiero M. Effects of Pre-surgical Vitamin D Supplementation and Ketogenic Diet in a Patient with Recurrent Breast Cancer. Anticancer Res. 2015;35(10):5525-32.

34. Tan-Shalaby JL, Carrick J, Edinger K, Genovese D, Liman AD, Passero VA, et al. Modified Atkins diet in advanced malignancies-final results of a safety and feasibility trial within the Veterans Affairs Pittsburgh Healthcare System. Nutr Metab. 2016;13:52. doi: https://doi.org/10.1186/s12986-0160113-y.
35. Klement RJ, Sweeney RA. Impact of a ketogenic diet intervention during radiotherapy on body composition: I. Initial clinical experience with six prospectively studied patients. BMC Res Notes. 2016; 9:143. doi: https://doi.org/10.1186/ s13104-016-1959-9.

36. Artzi M, Liberman G, Vaisman N, Bokstein F, Vitinshtein F, Aizenstein $\mathrm{O}$, et al. Changes in cerebral metabolism during ketogenic diet in patients with primary brain tumors: ${ }^{1} \mathrm{H}-\mathrm{MRS}$ study. J Neurooncol. 2017;132(2):267-75. doi: https://doi. org/10.1007/s11060-016-2364-x.

37. Tóth C, Clemens Z. Treatment of Rectal Cancer with the Paleolithic Ketogenic Diet: A 24-months Follow-up. Am J Med Case Reports. 2017;5(8):205-16. doi: https://doi. org/10.12691/ajmcr-5-8-3.

38. Cohen CW, Fontaine KR, Arend RC, Alvarez RD, Leath CA III, Huh WK, et al. A Ketogenic diet reduces central obesity and serum insulin in women with ovarian or endometrial cancer. J Nutr. 2018;148(8):1253-60. doi: https://doi. org/10.1093/jn/nxy119.

39. de Groot S, Lugtenberg RT, Cohen D, Welters MJP, Ehsan I, et al. Fasting mimicking diet as an adjunct to neoadjuvant chemotherapy for breast cancer in the multicentre randomized phase 2 DIRECT trial. Nat Commun. 2020; 3083. https:// doi.org/10.1038/s41467-020-16138-3. 